\title{
Artifacts Introduced Through the Hough Transform in EBSD
}

\author{
Xiaodong Tao and Alwyn Eades
}

\section{Department of Material Science and Engineering, Lehigh University, Bethlehem, PA 18015}

EBSD (Electron back scatter diffraction) has experienced rapid development in recent years. The breakthrough in EBSD came mainly from the rapid and accurate automatic indexing of EBSD patterns, for which the use of the Hough transform is most important. Even though the Hough transform is simple, easy and robust, the standard Hough transform[1] can also introduce artifacts. Figure 1 is an EBSD pattern from a 304 stainless steel sample. Figure 2 is the Hough transform of figure 1 using no convolution mask. Figure 3 is an enlarged view of the rectangular box in figure 2 . The intensity discontinuities, which are obvious in figure 3 are artifacts produced by the standard Hough transform. This type of artifact is most obvious when $\theta$ is equal to $45^{\circ}$ or $135^{\circ}$. Unfortunately, the performance of a convolution with a butterfly mask does not guarantee the elimination of such discontinuities all the time. The software can be easily cheated into finding a false band in the EBSD pattern.

The principle of the Hough transform is that the intensity at a point in the Hough space represents the intensity along a line in the original image. The coordinates in Hough space are usually taken as $\theta$ and $\rho$. Thus a point $\theta, \rho$ in the Hough transform represents a line at an angle $\theta$ and at a distance $\rho$ from the center of the original pattern. The standard way to perform the Hough transform is to calculate its value on a uniform grid. In another word it is evaluated for equal steps in angle and equal steps in $\rho$. The choice of equal steps in $\rho$ corresponds to evaluating the intensity along lines that have the same spacing independent of the angle of the line. Pixels in the image are assigned to the lines by rounding $\rho$ to an integer. Since the spacing of the pixels perpendicular to a particular line is not (in general) commensurate with the integer spacing of the lines, different lines (of the same parallel set) sample different numbers of pixels in the pattern. The effect is particularly dramatic at $45^{\circ}$ (and $135^{\circ}$ ) since, as shown in figures 4 and 5, some of the lines sample two rows of pixels whereas others sample only one. This is the origin of the artifacts shown in figure 3 .

We implemented and tested two methods that can fully remove these artifacts. "Nearest to the line". The principle of this method can be illustrated with reference to figures 5 , for the $45^{\circ}$ case. Some of the lines have twice as many pixels contributing to them as other lines. One way to correct the problem is simply to count the pixels that lie closest to the line and discard the pixels that are further away. This will remove the artifacts but does so at the expense of discarding data. "Weighting the points". The pixels given as triangles in figure 6 lie between two of the $45^{\circ}$ lines. In this method the intensities of these pixels will be added - with a suitable weighting factor - to both lines. The weighting will be larger the closer the pixel is to the line. Pixels that lie exactly on a line contribute to that line only. This method has the advantage that it uses all the data but still gives values in the Hough plot that correctly represent the intensities in the lines in the pattern.

The "nearest" method is simple and is readily compatible with the current commercial software. It is quite suitable for normal orientation mapping. Since part of our work involves detecting very small shifts in the bands, we use the "weighting" method which we expect to give the most accurate location of the peak in the Hough transform [2]. Another form of Hough transform[3], which uses the slope and intercept of lines as parameters, also has the potential to remove the artifacts. [4]. 


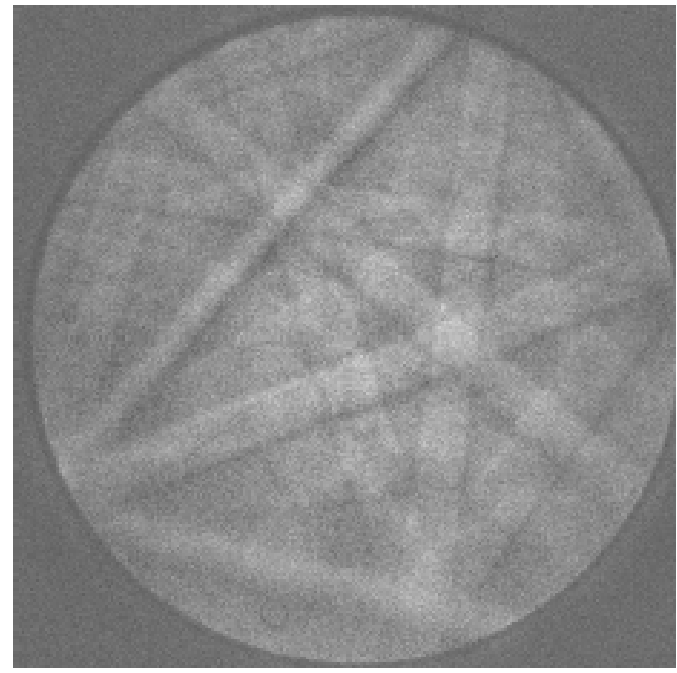

Figure 1. An EBSD pattern from 304 Stainless steel. The TSL OIM system and the FEI XL30 SEM were used.

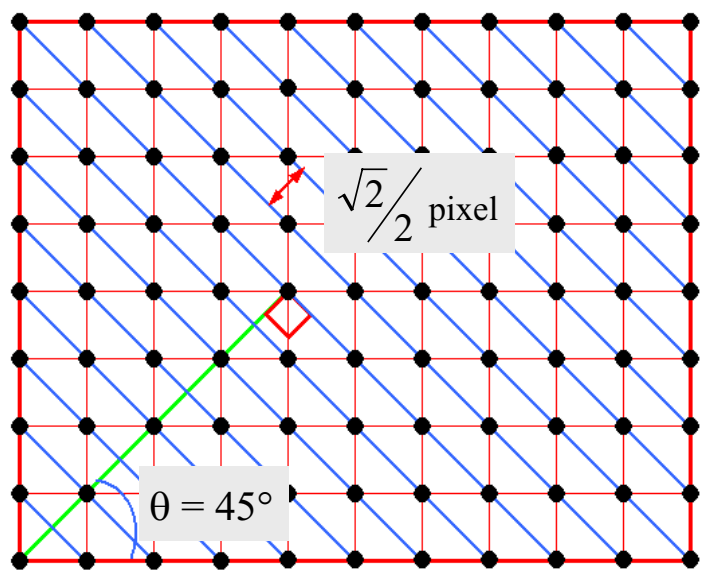

Figure 4. At $45^{\circ}$ the separation of the pixels is $\sqrt{2} / 2$ pixel

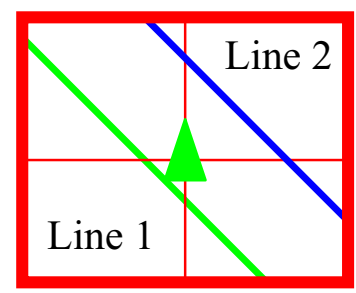

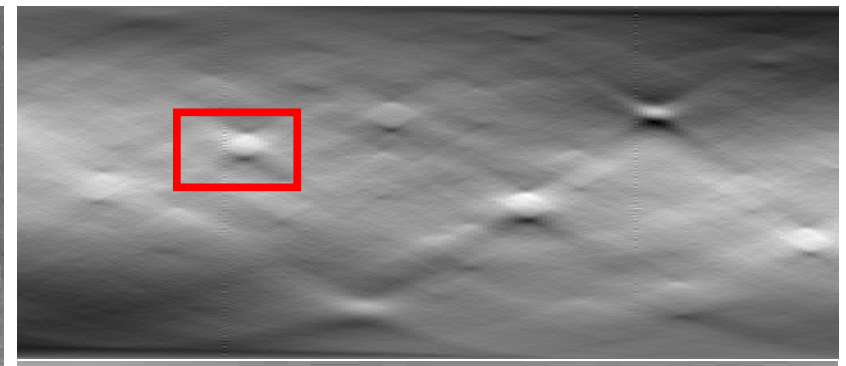

Figure 2

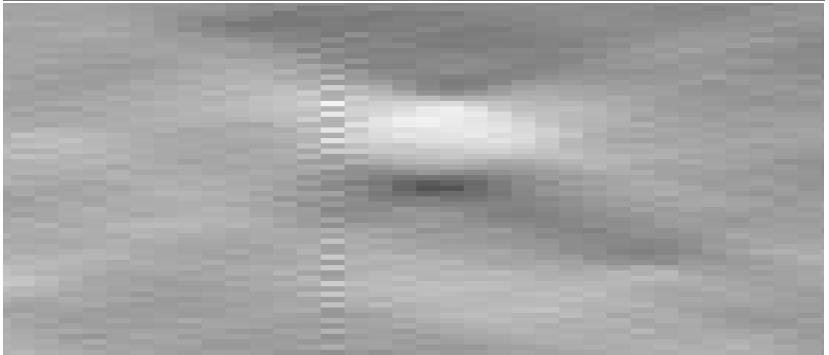

Figure 3

Figure 2. The Hough transform of figure 1, using no convolution mask. Figure 3. Enlarged view of the box in figure 2 .

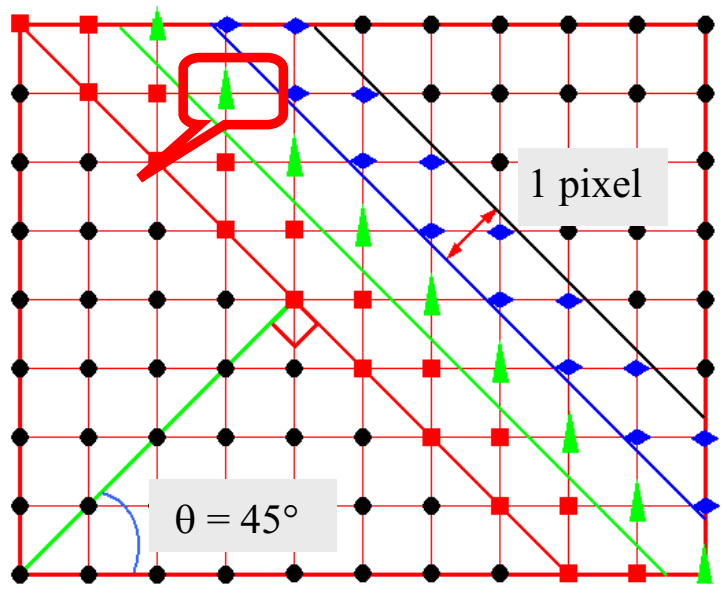

Figure 5. The Standard Hough transform samples the image space using equal steps of 1 pixel. As a result, some lines sample twice as many pixels as others.

Figure 6. Illustration of the "Weighting the points" method. The intensity of the pixel is distributed more to line 1 than to line 2 , according to its distance from the lines.

\section{References:}

[1] N.C. Krieger Lassen, D.J.Jensen and K.Conradson, Scanning Microscopy, 6 (1992) 115-121.

[2] Xiaodong Tao and Alwyn Eades, Microscopy and Microanalysis 7, (Suppl 2)(2001) 366-367.

[3] S. Krämer and J. Mayer, Journal of Microscopy, 194 (1999), 2-11.

[4] We are grateful to TSL for allowing us to use their source code.

Support from DOE, under grant DE-FG02-00ER45819, is gratefully acknowledged. 